\title{
Fundamental study of basal physical fitness and activities of daily living for the aged in relation to indoor horse riding exercise
}

\author{
Chang Ho Yu ${ }^{\mathrm{a}}$, Ui Ryeong Kim ${ }^{\mathrm{b}}$ and Tae Kyu Kwon ${ }^{\mathrm{a}, *}$ \\ ${ }^{a}$ Division of Biomedical Engineering, Chonbuk National University, Deokjin-Dong 1-Ga, Jeonju, \\ Jeonbuk 561-756, South Korea \\ ${ }^{b}$ Department of Healthcare Engineering, Chonbuk National University, Deokjin-Dong 1-Ga, Jeonju, \\ Jeonbuk 561-756, South Korea
}

\begin{abstract}
In this study, we assessed indoor horse riding exercise's effects on basal physical exercise and activities of daily living (ADL) function using horse riding equipment, involving elderly test subjects (in their sixties). The participants were 20 people with no impediment to activity. They participated in experiments that lasted $60 \mathrm{~min}$ per day, 3 days per week, over 8 weeks, using the "SRider" (Rider Co. and Chonbuk National University Korea).We measured trunk flexion, sit-up, wholebody reaction, leg strength, and maximal oxygen uptake as basal physical fitness parameters. Also, 3-m gait, single stance with eyes open, and single stance with eyes closed, as ADL functions, were estimated once per month. The leg strength and whole body reaction result were significantly higher than before the exercise program. Moreover, the results of the 3-m walking ability alone increased significantly among the ADL functions. These findings indicate that the horse riding exercise may activate continuous muscular contraction, maintaining the tonus of the muscles. The continuous movement of horse riding could be lead to isometric muscle contraction in the lower limbs. These results suggest that the horse riding exercise develops muscle power and muscle reactions with exercise.
\end{abstract}

Keywords: Horse riding, exercise effect, exercise instrument for indoor, the aged

\section{Introduction}

As a result of life-span prolongation due to economic and medical technology development, our country has become "an aging society." As of 2013, the population of those aged over 65 years old was 5.89 million persons and accounted for $11.8 \%$ of the total population. This trend is increasing every year [1]. In the aged, physiological and functional impairment are increased by aging and the functions required for the activities of daily living (ADL) decline [2]. Moreover, especially with falls, the elderly human body may be damaged readily $[3,4]$. Regular exercise for preventing such damage is an important element for promoting general physical function in the aged [5]. Additionally, as the muscular sense of balance is enhanced through exercise, stable physical standing function can be im-

\footnotetext{
${ }^{*}$ Corresponding author: Tae Kyu Kwon, Division of Biomedical Engineering, Chonbuk National University, Deokjin-Dong 1-Ga, Jeonju, Jeonbuk 561-756, South Korea. Tel.: +82-63-270-4066; Fax: +82-63-270-2247; E-mail: kwon10@jbnu.ac.kr.
} 
proved this is effective for reducing a risk of a fall [6,7]. However, as outdoor activity or exercise in the elderly then exposes them to diverse issues that may cause physical injury, even such exercise may involve high risks $[8,9]$. For this reason, regular indoor exercise, rather than outdoor exercise, is typically recommended for the elderly. Such indoor exercises include weight-lifting, swimming, cycling, and horse riding. Recently, horse riding has been spot-lighted because it provides users with interest and has the effect of a total-body workout [10].

Horse riding exercise is known as a total-body workout, having an outstanding exercise effect; furthermore, its rehabilitation effects are also excellent [11]. The principle of horse-riding exercise is a process of maintaining a stance repeatedly by matching the horse's movements and this is effective for enhancing postural balancing ability, muscular strength, and flexibility [12]. The 3D movement of the horse is known to be effective in recovering deteriorated function by activating muscles and joints, stimulating even core muscles that are usually not used by a horse rider and promoting blood circulation [13]. Horse-riding exercises produce an exercise effect similar to general exercise of low strength and ideal for the aged with knee and waist pain [14]. Additionally, as studies have shown positive correlations between horse riding exercise and muscular endurance, speed, coordination, flexibility, balance, and aerobic and anaerobic capacity, the effects of horse-riding exercises have been demonstrated [15].

Horse-riding exercises by actually riding a horse have not yet been popularized in our country, due to temporal, spatial, cost limitations. For these reasons, much horse riding exercise equipment has been developed and efforts to determine the exercise effects of horse riding exercise equipment have been made. It has been shown that horse riding exercises using a horse riding simulator is appropriate for the purpose of improving activities of daily living and preventing falls in the elderly [11]. Indoor horse riding exercise is positively effective for preventing falls by improving balance not only for the aged with dementia but also for the general aged [16]. However, as most of the studies on horse riding exercise were performed actually using horses, or simulator, they have not examined indoor exercise equipment. Additionally, many of the studies on the exercise effect of existing indoor horse riding exercises have targeted young adults or even athletes and studies on aged subjects are inadequate.

Thus, in this study, indoor horse riding as an exercise method that could be applied to the aged who had difficulty in outdoor exercise or autonomous exercise was examined. For this objective, the effects of horse riding exercise were evaluated by measuring and analyzing basal physical fitness parameters and ADL function in the aged based on horse riding exercises using indoor horse riding exercise equipment.

\section{Experimental methods}

\subsection{Subjects}

In this study, an exercise effect was intended to be verified objectively through evaluation of body composition and physical fitness based on horse riding exercise by targeting the male, female elderly subjects in their $60 \mathrm{~s}$. By random sampling 60 elderly aged over 60 years old who had no medical history or exercise injury in the past 6 months and had no experience of aerobic or anaerobic exercises in the past 3 months, were evaluated in terms of their basal physical fitness level. Among them, 20 subjects whose basal physical fitness level was within the average $\pm 10 \%$ were selected for this study. Prior to the beginning of the examination, conforming to the Declaration of Helsinki (1964), written informed consent was obtained from all subjects. 


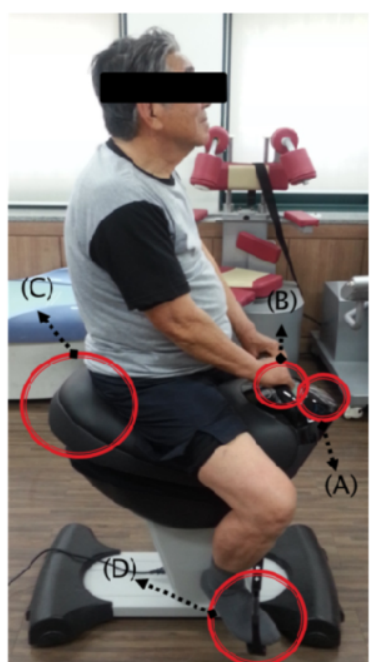

(a)

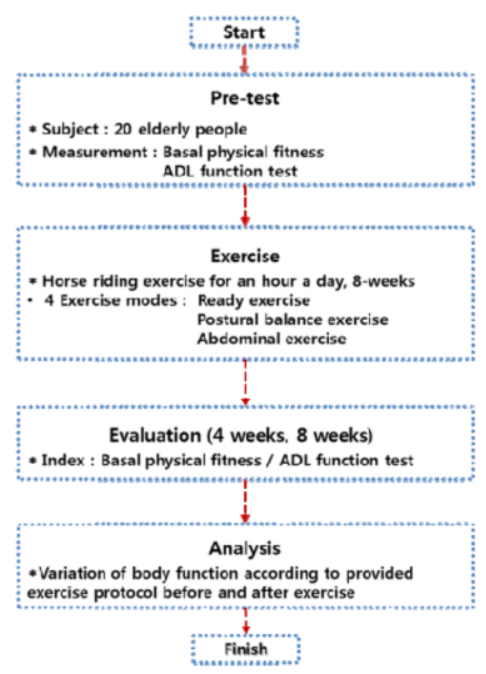

(b)

Fig. 1. (a) The "SRider" horse riding exercise instrument (Rider Co. and Chonbuk National University, Korea): (A) Control panel (B) Handle (C) Saddle (D) Pedal, (b) Block diagram of the horse-riding exercise program.

\subsection{Experimental equipment}

The "SRider" (Rider Co. and Chonbuk National University, Korea) used for the experiment is an indoor horse riding exercise instrument. As shown in Figure 1(a), its shape simulates horse riding and exercise strength is controlled by adjusting the speed and range of saddle movement.

\subsection{Procedure and method of experiment}

In this study, to evaluate the effects of horse riding exercise in the aged, basal physical fitness and ADL function before and after the horse riding exercise program were measured. Exercise progressed using the horse riding equipment for 8 weeks based on $1 \mathrm{~h}$ per day, 3 days per week, and to eliminate error due to differing postures among the test subjects during the horse-riding exercise, the exercise session was monitored so that subjects all maintained a constant posture. Additionally, the objective and methods of the experiment were explained to the subjects so that they may become familiar with the objective and method. Figure 1(b) shows a block diagram of the horse-riding exercise program.

\subsection{Movement of horse riding exercise equipment and posture}

The SRider provides movement by extensively shaking the whole body in every direction and a rotating movement based on the abdomen at the same time. Additionally, in the posture of the horse riding exercise, test subjects were directed to maintain their posture, flattening the waist, at $90^{\circ}$, and to turn their eyes to the front during the horse-riding exercise. To eliminate postural error among test subjects, they were directed to maintain the same stance (posture). 


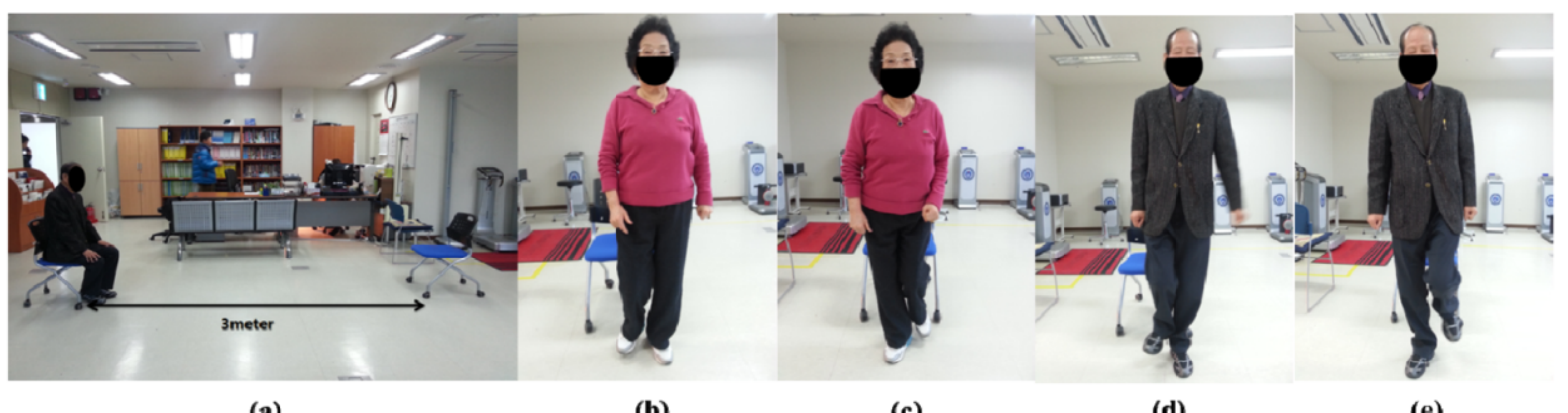

(a)

(b)

(c)

(d)

(e)

Fig. 2. ADL function tests: (a) 3-m walking (b) Single stance with eyes open and right leg lifted (c) Single stance with eyes open and left leg lifted (d) Single stance with eyes closed and right leg lifted (e) Single stance with eyes closed and left leg lifted.

\subsection{Measurement of basal physical fitness and ADL function}

In this study, to evaluate physical fitness in the aged, an evaluation of basal physical fitness and ADL function was performed. The basal physical fitness evaluation comprised bending the back forward (flexibility), sit-ups (muscular endurance), total-body reaction test (speed), muscular strength of the lower extremities test, and cycle ergometer measurements (cardiovascular endurance). Additionally, to evaluate ADL function, 3-m walking ability, single stance standing with eyes opened and one leg lifted, single stance standing with eyes closed and one leg lifted were measured. The 3-m walking ability was evaluated by measuring the time to walk from one chair to another at an ordinary walking speed after standing from one chair followed by placing two chairs at both sides at a spacing of $3 \mathrm{~m}$. Single stance standing with eyes opened and one leg lifted and single stance standing with eyes closed were measured once each based on the right leg and left leg, respectively.

\subsection{Data analysis}

To verify the validity of the measured data, the data of the basal physical fitness evaluation and ADL function were analyzed using the SPSS software (ver. 18.0). In the case of the basal physical fitness evaluation data, mean values and standard deviations of leg muscular strength, muscular endurance, speed, flexibility, power, and aerobic function were estimated and to verify an effect before and after exercise in each basal physical fitness parameter and ADL function item, the paired t-test was used. The significant levels were $\mathrm{p}<0.05$ and $\mathrm{p}<0.01$.

\section{Results and discussion}

In this study, the effects of indoor horse riding exercise on basal physical fitness and ADL function in the elderly were examined. An experiment was performed using general horse-riding exercise equipment that is commonly available locally. Variation in basal physical fitness and ADL function before and after the exercise program was analyzed. 


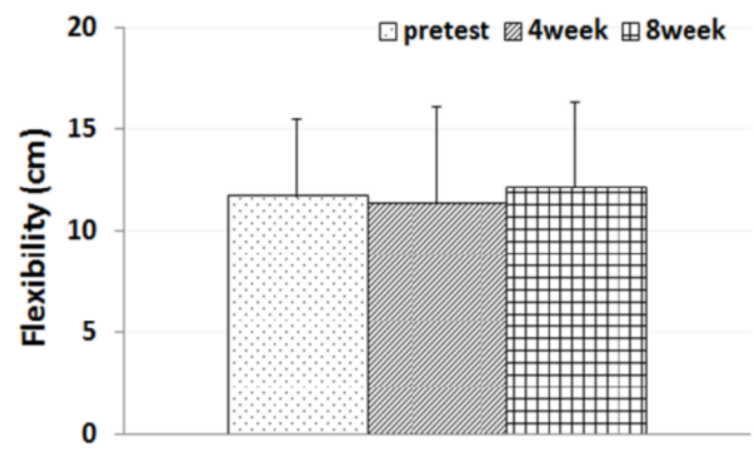

(a)

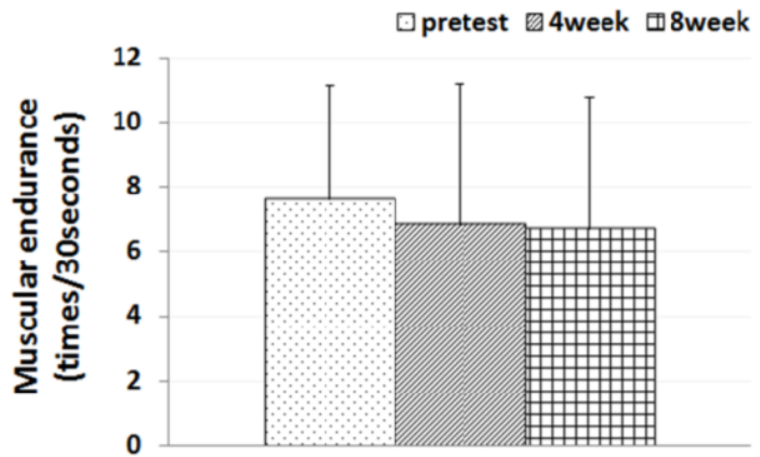

(b)

Fig. 3. Basal physical fitness before and after exercise in the aged subjects: (a) Variation in flexibility (mean $\pm \mathrm{SD},{ }^{*} \mathrm{p}<0.05$ ) (b) Variation in muscular endurance (mean $\pm \mathrm{SD}, * \mathrm{p}<0.05)$.

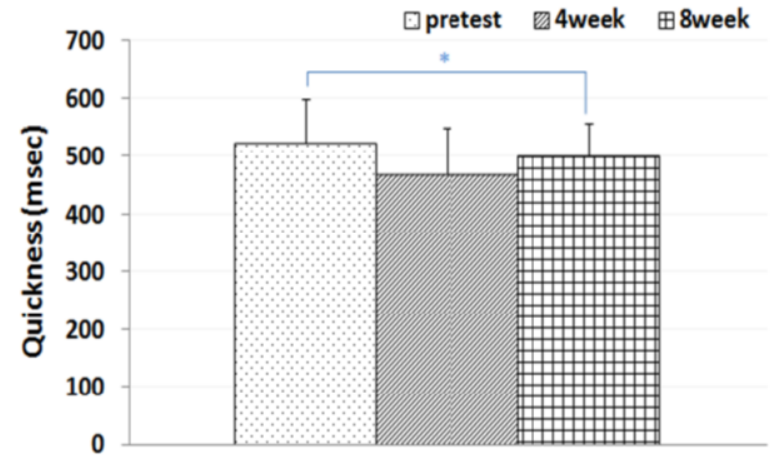

(a)

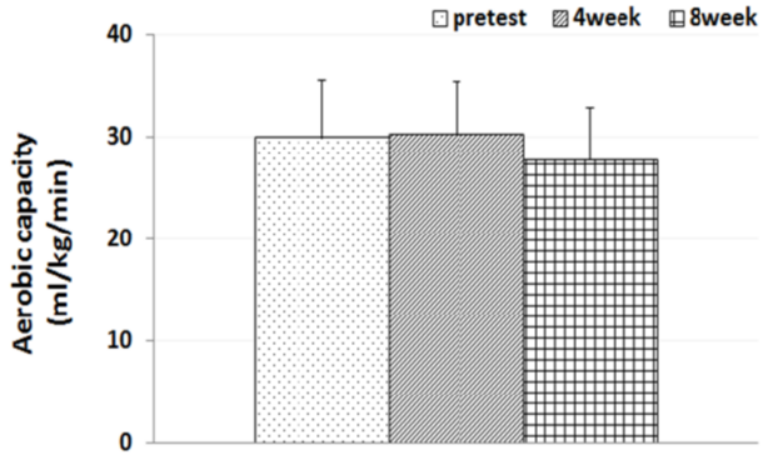

(b)

Fig. 4. Basal physical fitness for before and after exercise in the aged subjects: (a) Variation in speed (mean $\pm \mathrm{SD},{ }^{*} \mathrm{p}<0.05$ ) (b) Variation in aerobic capacity (mean $\pm \mathrm{SD},{ }^{*} \mathrm{p}<0.05$ ).

\subsection{Variation in basal physical fitness as a result of horse-riding exercise program}

Variation in basal physical fitness before and after the exercise program using indoor horse-riding equipment was measured. Figure 3 shows (a) flexibility and (b) muscular endurance among basal physical fitness evaluation elements before and after exercise. To measure variation in flexibility, a bending backwards and forwards test was performed. The value was increased but not statistically significantly so. The reason why may be attributable to the fact that the exercise motion in the horseriding exercise took place within the range of joint motion in the test subjects. To measure variation in muscular endurance, sit-ups were performed for $30 \mathrm{~s}$ and the frequency showed a tendency to decrease

Figure 4 shows (a) agility and (b) aerobic ability among the basal physical fitness evaluation elements before and after exercise. Agility is based on physiological elements, including the relationship between nerves, muscles, and the speed of muscular contraction. To measure variation in agility, a total-body reaction test was used and it was confirmed that the reaction time was shortened significantly. This indicates that agility was enhanced and it was correlated with enhanced muscular strength of the lower extremities too. It was considered that sustained muscular contraction associated with horse-riding exercise would promote muscular strength by maintaining muscle tone and increasing 
blood flow to muscles and that this would enhance agility, the foundation of the relationship between nerves and muscular strength. Additionally, it was considered that agility had a relationship with speed of muscular contraction and as muscular strength was increased, speed of muscular contraction was accelerated too. To evaluate aerobic ability, maximal oxygen intake was measured using a cycle ergometer. As a result, it was confirmed that maximal oxygen intake after exercise was decreased versus that before exercise.

Finally, Figure 5 shows leg muscle strength among the basal physical fitness evaluation elements before and after the horse-riding exercise program. Leg muscular strength refers to the isometric maximal muscular strength of the lower extremities. As a result of the experiment, right leg muscular strength and left leg muscular strength were increased significantly. These results indicate that muscular strength was enhanced by the horse-riding exercise. The femoral region muscles of the lower extremities are persistently contracted to maintain body balance [17]. This is similar to the result that enhancement of muscular strength in dementia patients is effective in reducing the risk of falls [6].

\subsection{Variation in $A D L$ function as a result of the horse-riding exercise program}

In this study, not only basal physical fitness but also ADL function was evaluated in the elderly. Figure 6 shows the maintaining time of a single stance on the left or right leg after providing visual information ((a) right, (b) left). As a result, for a single stance on the right or left leg, the time was increased in both cases but not statistically significantly so.

Figure 7 shows the maintaining time of a single stance on one leg without visual information before and after the horse-riding exercise ((a) right, (b) left) into statistically significant difference was found. Proprioceptors generally play a significant role based on coordinated action between muscular strength of the trunk and lower extremities and muscular endurance in the sense of balance. As a result of this study, it is considered that a positive result in enhancing the sense of balance was not seen in terms of muscular strength of the trunk, although the muscular strength of the lower extremities was increased significantly.

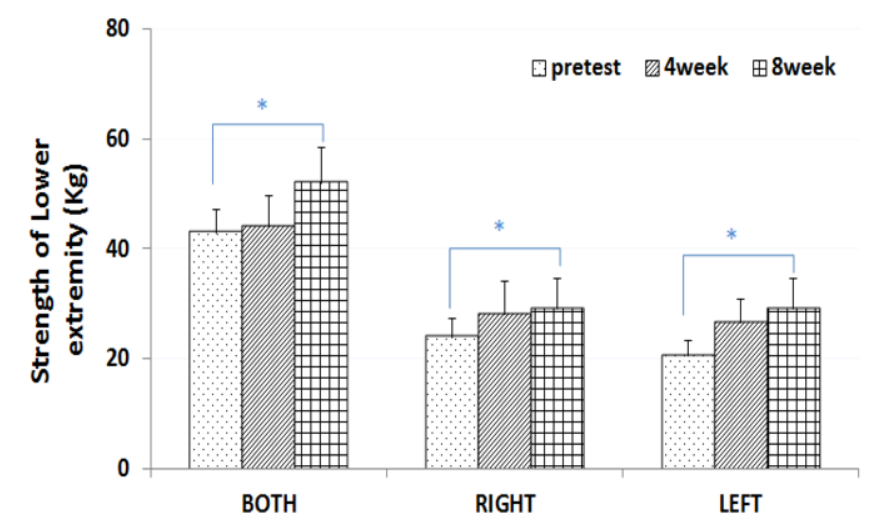

Fig. 5. Basal physical fitness before and after exercise in the elderly subjects: variation in leg muscle power (mean $\pm \operatorname{SD},{ }^{*} \mathrm{p}$ $<0.05)$. 


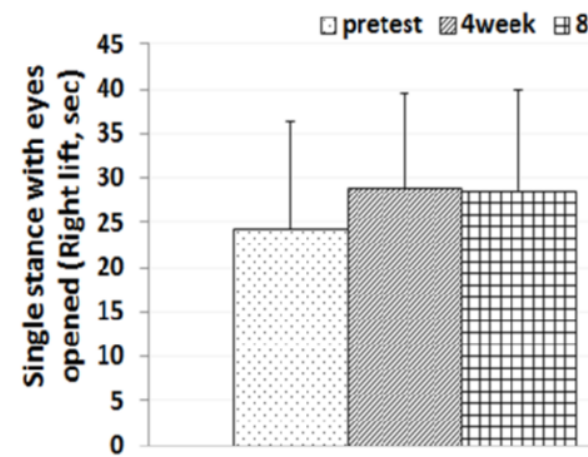

(a)

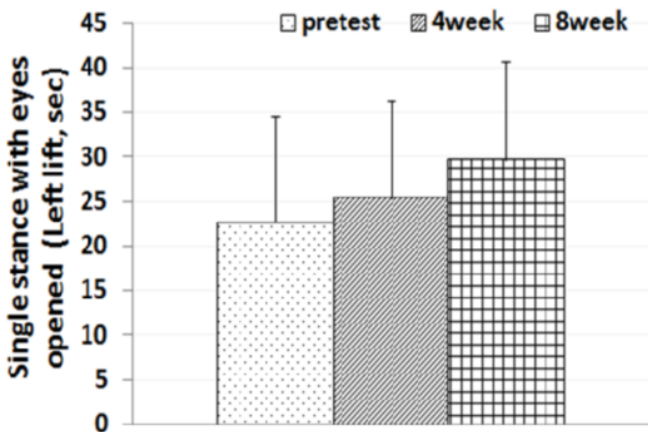

(b)

Fig. 6. ADL function test before and after exercise program in the elderly: (a) Time variation of single stance with eyes open and right leg lifted (mean $\left.\pm \mathrm{SD},{ }^{*} \mathrm{p}<0.05\right)$ (b) Time variation of single stance with eyes open and left leg lifted (mean $\pm \mathrm{SD}$, $* \mathrm{p}<0.05)$.

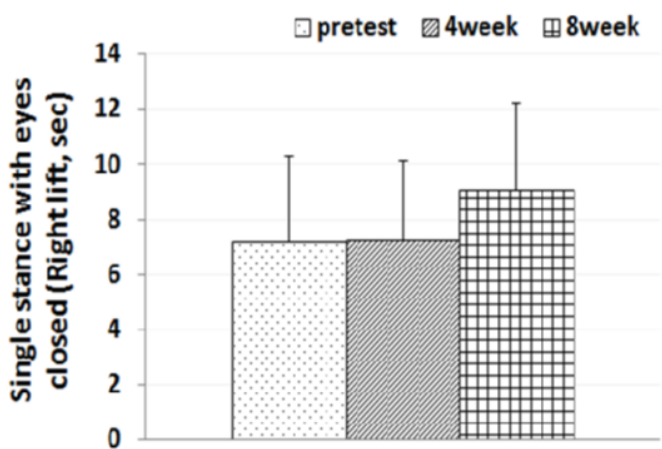

(a)

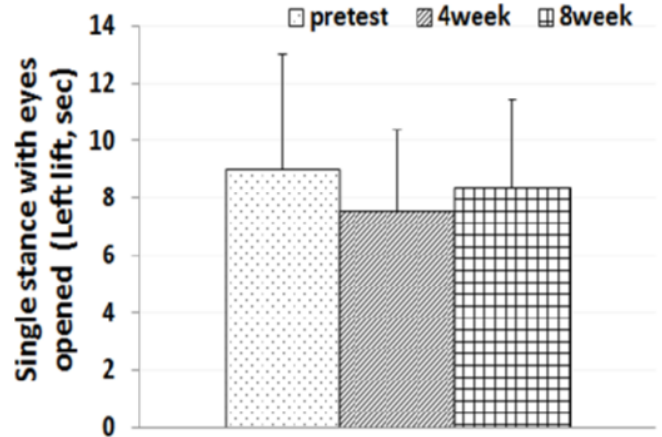

(b)

Fig. 7. ADL function test before and after exercise program in the elderly: (a) Time variation of single stance with eyes closed and right leg lifted (mean $\pm \mathrm{SD}, * \mathrm{p}<0.05$ ) (b) Time variation of single stance with eyes closed and left leg lifted (mean $\pm \mathrm{SD},{ }^{*} \mathrm{p}<0.05$ ).

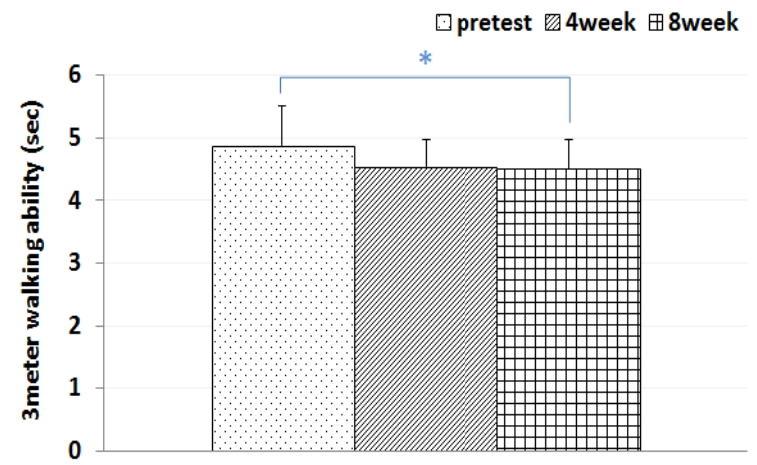

Fig. 8. Variation in 3-m walking ability before and after exercise as a test of ADL function in the aged (mean $\pm \mathrm{SD}$, $* \mathrm{p}<0.05)$. 
Figure 8 shows the time required for sitting down again after walking $3 \mathrm{~m}$ following standing up from a sitting position. It was confirmed that as walking ability time was significantly decreased, walking ability was enhanced. It is considered that the reason is that the muscular reaction time at the moment of standing up from the chair and then sitting down on it and the walking speed increased due to muscular strength being increased as a result of the lower extremities muscular strength and agility being increased due to the horse-riding exercise program.

\section{Conclusion}

In this study, an indoor horse riding exercise was suggested as an exercise method applicable to the aged who may have difficulty participating in outdoor exercises or autonomous exercises. By using indoor horse riding equipment, the effects on basal physical fitness and ADL function in the elderly were analyzed. The following conclusions can be drawn.

In a preceding study, it was reported that horse-riding exercises shows promoted a sense of balance, muscular strength, and flexibility. In this study, similar results in terms of enhancing muscular strength of the lower extremities was obtained, although in terms of flexibility, no significant result was obtained.

Horse-riding exercise showed a positive effect in reinforcing muscular strength in the lower extremities, because the femoral region of the lower extremities is contracted persistently to maintain body balance (right: increase by $17 \%$, right: left: increase by $29 \%$, both: increase by $17 \%$ ). Enhancement of muscular strength in the lower extremities resulted in significant promotion of agility too (reaction time reduced by $4.3 \%$ ) and it is considered that this agility increase will eventually lead to the strengthening of muscular reactions.

It is considered that enhancing these two physical fitness elements leads to the promotion of walking ability in the aged. Thus, indoor horse-riding exercises may be helpful to the aged for enhancing muscular strength of lower extremities and may provide a positive exercise effect as an indoor exercise suitable for patients and the disabled who have difficulties in outdoor activities generally, as well as the aged.

\section{Acknowledgment}

This work was supported by Industrial-Complex Cluster Program of the KICOX (Korea Industrial Complex Corp) grant funded by the Korea government Ministry of Trade, Industry and Energy (1415130494) and partially supported by the Sports Promotion Fund of Seoul Olympic Sports Promotion Foundation from Ministry of Culture, Sports and Tourism.

\section{References}

[1] S.G. Kim, G.C. Yuk and H.B. Gak, Effects of the horse riding simulator and ball exercises on balance of the elderly, Journal of Physical Therapy Science 25 (2013), 1425-1428.

[2] S.S. Bae and R.J. Park, Rehabilitation of geriatric patient, The Korean Society of Physical Therapy 2 (1990), 95-101.

[3] J.W. Kim, H.Y. Jeong, H.H. Kim, Y.R. Kwon, G.M. Eom, J.W. Lee, K.S. Kim, J.H. Jun and B.K. Park, Comparison of reaction times of ankle joint muscles in the elderly men and women, Journal of Biomedical Engineering Research 33 (2012), 163-168. 
[4] H.J. So, K.Y. Kwak, S.H. Kim, Y.S. Yang, N.G. Kim and D.W. Kim, The analysis of GRF and joint angles of young and older adult by vibration stimulation on the ankle-joint in stair-descent activity, Journal of Biomedical Engineering Research 32 (2011), 61-73.

[5] E.B. Larson and L. Wang, Exercise, aging, and Alzheimer disease, Alzheimer Disease \& Associated Disorders 18 (2004), 54-56.

[6] R.B. Flannery, Treating learned helplessness in the elderly dementia patient: preliminary inquiry, American Journal of Alzheimer's Disease and Other Dementias 262 (2002), 2551-2556.

[7] S.J. Hwang, J.S. Son, J.Y. Kim, H.D. Kim, D.H. Lim and Y.H. Kim, Analysis of joint movements and changed of muscle length during STS (sit-to-stand) at various sitting heights in the Korean elderly's daily life, Journal of Biomedical Engineering Research 29 (2008), 484-492.

[8] A.S. Howe and B.P. Boden, Heat-related illness in athletes, The American Journal of Sports Medicine 35 (2007), 13841395.

[9] U. Bjornstig, J. Bjornstig and A. Dahlgren, Slipping on ice and snow-elderly women and young men are typical victims, Accident Analysis \& Prevention 29 (1997), 211-215.

[10] S.K. Lee and J.H. Jeong, The effects of indoor horseback-riding exercise on health-related fitness, serum lipids, and defecation satisfaction of female collegiate students, Korea Sport Research 16 (2005), 153-160.

[11] D.B. Bertoti, Effect of therputic horseback riding on posture in children with cerebral palsy, Journal of Physical Therapy 68 (1998), 1505-1512.

[12] W.Y. Oh, C.C. Ryew, J.H. Kim and S.H. Hyun, Kinematic analysis of horse-riding posture according to skill levels during rising trot with JeJu-horse, Korean Journal of Sport Biomechanics 19 (2009), 467-479.

[13] G.R. Park and S.J. Kwon, An influence of horse riding on gross motor skill development in mentally handicapped students, The Korea Journal of Sports Science 20 (2011), 1003-1009.

[14] H. Yoshiyuki, N. Masaru, B. Gustavo, S. Youichi, O. Takahisa and S. Yuzo, Effects of daily mechanical horseback riding on insulin sensitivity and resting metabolism In middle-aged type 2 diabetes mellitus patients, Nagoya Journal of Medical Science 72 (2010), 129-137.

[15] H.A. Alfredson, G. Hedberg, E. Bergstrome, P. Nordstrom and R. Lorentzon, High thigh muscle strength but not bone mass in young horseback-riding female, Calcified Tissue International 62 (1998), 497-501.

[16] D.H. Kim, S.J. Kim, S.S. Bae and K. Kim, The effect of indoor horseback-riding machine on the balance of the elderly with dementia, The Korean Society of Physical Medicine 3 (2008), 235-246.

[17] S.R. Kang, C.H. Yu, G.Y. Jeong, D.A. Moon, S.Y. Park, J.J. Kim and T.K. Kwon, Analysis of the indoor horse riding exercise equipment on the young people, International Journal of Precision Engineering and Manufacturing 14 (2013), 1471-1478. 